\title{
MODEL REDUCTION USING NEURAL NETWORKS APPLIED TO THE MODELING OF INTEGRATED URBAN WASTEWATER SYSTEMS
}

\author{
Botond Ráduly and \\ Andrea G. Capodaglio \\ Dept. of Hydraulic\&Environ. \\ Engineering \\ University of Pavia \\ Via Ferrata 1, 27100 Pavia \\ PV, Italy \\ E-mail: raduly.botond@unipv.it
}

\author{
Erik Lindblom \\ Institute of Environment \& Resources \\ Technical University of Denmark \\ Bygningstorvet, Building 115 \\ DK-2800 Kgs. Lyngby, Denmark \\ E-mail: eul@er.dtu.dk
}

\author{
Krist V. Gernaey \\ Dept. of Chemical Engineering \\ Technical University of Denmark \\ Building 229, DK-2800 Kgs. Lyngby \\ Denmark \\ E-mail: kvg@kt.dtu.dk
}

\begin{abstract}
KEYWORDS
Integrated modeling, artificial neural networks, simulation speed, integrated urban wastewater system simulation, wastewater treatment plant modeling
\end{abstract}

\begin{abstract}
Simulation of the integrated urban wastewater system is a computationally-demanding task, and performing long-term simulations consequently takes a considerable time. Reduction of simulation times can be achieved by speeding up one or more of the submodels of the integrated system. In this paper the use of a fast neural network model instead of the mechanistic model of the wastewater treatment plant (WWTP) is proposed for this purpose. The neural network is trained on a sequence of treatment plant input/output data generated by the mechanistic model of the WWTP, i.e. it is a reduced model of the original WWTP model. As a result of model substitution a reduction of the simulation time by a factor of 23 was achieved. The results presented in this paper show that the errors introduced by the WWTP model substitution are of an acceptable level, confirming the practical usefulness of the proposed method.
\end{abstract}

\section{INTRODUCTION}

The integrated urban wastewater system (UWWS) typically consists of the sewer system, the WWTP and the receiving water body (usually a river). Integrated modeling of the UWWS supposes the simultaneous simulation of the models of each subsystem (Schütze et al. 2002). Simulation studies performed with such integrated models are very useful in assessing the impact of sewer overflows and WWTP performance on the quality of receiving waters, enabling the evaluation of the effectiveness of the measures taken to reduce the impact.

The submodels of the UWWS are often mechanistic models, consisting of a set of ordinary differential equations (ODEs) that describe the physical, chemical and biological processes occurring in the system. As all of these submodels are already of considerable complexity, coupling them together in an integrated model leads to even more complex large scale models.
Such integrated models might be less adequate to study the interplay between the components of the system. As one may get lost in model details, the perspectives of the holistic approach, which is the aim of integrated models, may vanish among the numerous parameters.

The second problem with the high level of complexity is that it makes these models rather slow. Low simulation speeds occur mainly due to the use of mechanistic WWTP models. Such WWTP models are rather stiff systems, with a broad range of time constants, necessitating small integration steps and consequently considerable CPU time. A state-of-the-art WWTP model contains several control loops and discrete sensors, that turns it into a hybrid system; the stiff numerical solvers, designed for fast integration of stiff systems, work very poorly for hybrid systems (Copp 2002). Thus the simulation of the integrated UWWS is a modeling application often limited by the long simulation time of the mechanistic WWTP model.

In order to decrease simulation times, different model reduction strategies might be applied. Some of these imply the use of simplified mechanistic models (Butler and Schütze 2005); other strategies obtain reduced models by boundary allocation (Vanrolleghem et al. 2005); replacement with empirical models is another viable method that may lead to significantly increased simulation speed (Ráduly 2005).

In this study the use of an artificial neural network (ANN) is proposed to replace the mechanistic WWTP model, identified to be the main contributor to the computational burden. ANN models are completely data-driven, meaning that they can be built using only input/output data of the modeled system, i.e. the ANN model can be trained using data generated with the mechanistic WWTP model. Although ANNs might have a rather complex structure, they are based on simple mathematics and thus they are significantly faster than the corresponding mechanistic models. One of the drawbacks of such model substitution is the black-box nature of the ANNs: although an ANN may perform correct predictions, the input-output relationships remain practically hidden. It also means that if any change is done to the mechanistic model, the ANN has to be trained again. This disadvantage can be somehow 
reduced if short ANN training times can be achieved. The lack of transparency can also be tolerated in many modeling applications, e.g. if a full calibrated treatment plant model is replaced with the ANN and if the modeling objectives are at the integrated model level (e.g. studying the interaction between submodels). In such cases the use of an ANN model with no adjustable parameters might also be an advantage, as the modeler can focus on the main modeling goals instead of getting lost among the numerous model parameters.

\section{MATERIALS AND METHODS}

A model of a virtual urban wastewater system has been built, consisting of an influent model, a WWTP model and a river model. Converter (transformer) modules were used to "translate" the state variables of the different sub-models for the other sub-models. Two different configurations were tested: one consisting of only mechanistic models, and another one using an ANN model instead of the mechanistic model of the treatment plant (Fig. 1). The evaluation of the results includes the comparison of the river quality indicators downstream the WWTP outlet and the comparison of simulation speeds of the two different model configurations. If the final results (i.e. river quality parameters) obtained with the two model configurations show a high correlation, this would indicate that the prediction errors induced by the ANN are not significant at an integrated model scale. The mechanistic model results are used as reference in the comparison. The criteria for the model comparison are the monthly chemical oxygen demand (COD) loads to the river as well as the concentrations of dissolved oxygen (DO), ammonium $\left(\mathrm{NH}_{4}\right)$ and biochemical oxygen demand $\left(\mathrm{BOD}_{5}\right)$ in the river reaches downstream of the WWTP outlet.

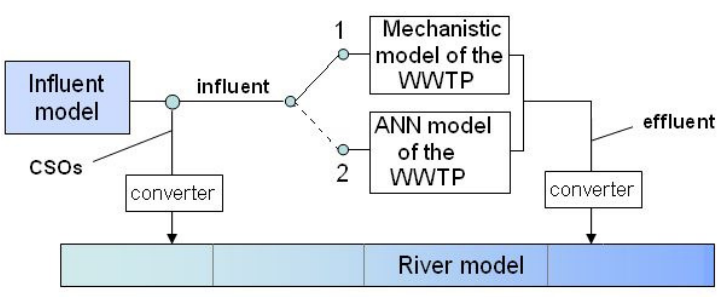

Figure 1. The Integrated Model Configuration. If the Switch is in Position 1, the Mechanistic WWTP Model is Used, in Position 2 the ANN Model is Used

\section{The Integrated UWWS Model}

The integrated model of the UWWS (Fig. 1) includes the following components: (1) an influent model which describes the catchment, the rainfall/runoff and the sewer processes; (2) a flow splitter, which limits the flow rate to the WWTP at $60000 \mathrm{~m}^{3} / \mathrm{d}$ (the rest of the wastewater is directed to the river as combined sewer overflows (CSOs); (3) a WWTP model, which describes the wastewater treatment processes; (4) a river model, which describes the flow and the conversion processes taking place in the river and finally (5) transformer modules, which enable the connection between the different sub-models (only 2 such modules are needed, as the influent model and the WWTP model are directly compatible). The different submodules will be introduced one by one in the following sections.

The output of the influent model is used as the WWTP model input. If the flow rate generated by the influent model exceeds $60000 \mathrm{~m}^{3} / \mathrm{d}$ (which is the maximum hydraulic capacity of the treatment plant, representing approx. three times its nominal flow), the flow splitter will direct the excess flow to the river (CSOs). Both the CSOs and the WWTP effluent variables are "translated" by transformer modules to be compatible with the river model state variables. The river model consists of several river segments. The CSOs and the WWTP effluent reach the river at two different points. The WWTP effluent emission point is located downstream relative to the CSOs.

For simplicity, a scenario with no CSOs has been chosen for this study. CSOs are important disturbances for the river. However, the aim of this study is testing the ANN model, which can be done without CSOs. The CSOs would indeed be identical for all scenarios, and their absence in the work presented in this paper will not impede the evaluation of the differences between the results of the two model configurations, as the differences are introduced by the ANN.

The methodology used for model comparison in this paper did not necessitate the simultaneous simulation of all UWWS submodels. The data sequences generated with the influent model were first obtained, and were then used in the subsequent simulation steps. In a similar way the WWTP simulations were done separately, and these results were finally used as input for the river model simulation.

All modeling work was done in the Matlab/Simulink modeling environment.

\section{The Influent Model}

Originally developed as an influent disturbance scenario generator to be included in future extension of the IWA/COST simulation benchmark plant (Copp 2002; Jeppsson et al. 2006), the influent model (see Gernaey et al. (2005, 2006) for details) generates WWTP influent concentration profiles that are compatible with the Activated Sludge Model No. 1 (ASM1; Henze et al. 1987). It includes simple phenomenological representations of the typical phenomena that are observed in full-scale WWTP influent flow rate, concentration and temperature data: (1) diurnal phenomena; (2) weekend effects (e.g. lower pollutant loads); (3) seasonal phenomena reflecting typical effects from the sewer system and urban drainage, (e.g. increased infiltration in the rainy season compared to the 
dry season); (4) holiday periods (e.g. with a lower average wastewater flow rate); (5) rain events.

\section{The WWTP model}

For the deterministic part of the treatment plant module, the ASM1 and a non-reactive Takács settler model (Takács et al. 1991) were used for simulating the activated sludge tanks and the secondary settler respectively. These models describe the physical, chemical and biological processes of an activated sludge WWTP using ordinary differential equations. The plant lay-out specifications of the Benchmark Simulation Model no. 1 (BSM1, Copp 2002) were chosen as a case study. The plant, a predenitrification system, is described in detail in Copp (2002). The differences, as compared to the original BSM1 specifications, consist of the cascade DO control and the modeling of temperature-dependency of the biochemical conversion processes.

The deterministic WWTP model contains, besides the algebraic equations, 135 ODEs and 4 control loops. The high number of differential equations and the additional system stiffness introduced by the control loops consequently leads to slow numerical processing and long simulation times.

\section{The river model}

A reduced version of the entire River Water Quality Model No. 1 (RWQM1) developed by the IWA task group (Reichert et al. 2001) is used to describe the physical, chemical and biological processes in the river. The states of this model are more like the ASM1 states, but some differences still remain. This is due to the fact that the full RWQM1 model has to describe more processes than the ASM1 model (e.g. algae growth). Hence transformer modules (Section 4.6.3.4) are needed to allow for the data flow between the ASM-based WWTP models and the RWQM1.

The river model implemented represents a $16 \mathrm{~km}$ long river stretch and is divided into 16 reaches $(1 \mathrm{~km}$ each). The overflows enter the river in reach no. 5, while the WWTP effluent enters in reach no. 7; the distance between the CSO structure and the WWTP is $2 \mathrm{~km}$. The base flow of the river is $64800 \mathrm{~m}^{3} / \mathrm{d}$, and increases constantly with $0.1728 \mathrm{~m}^{3} / \mathrm{m}$ river; thus the base flow at the WWTP outlet is $66010 \mathrm{~m}^{3} / \mathrm{d}$. This is about 3.5 times more than the nominal flow rate of the WWTP effluent, that is to say the WWTP discharges in a small river. During rain events the rainwater will enter the river only via the WWTP for the simulations reported in this paper; this means that at the maximum hydraulic capacity the flow rate of WWTP effluent is comparable to the river's flow rate. The river flow : WWTP flow ratio is quite extreme, but appropriate for this model comparison study. As the pollutants discharged by the WWTP will be only slightly diluted by the river water, the prediction errors introduced by the ANN model will be more visible when looking at the concentrations in the river.

\section{The transformer modules}

In order to perform simulations of the integrated wastewater system, state variables of sub-system models must be translated from one model to the other. The transformers used in this work translate ASM1 variables to RWQM1 variables and were presented by Benedetti et al. (2004).

\section{The ANN Model}

A neural network model consists of a set of parallel interconnected simple computational units, called neurons. Such a model resembles the brain in two aspects: (1) knowledge is acquired by the neurons through a learning process and (2) inter-neuron connection strengths, known as synaptic weights, are used to store the knowledge (Haykin 1994). The learning process encompasses the adjustment of the weights of each processing unit, and can be seen as teaching the network to yield a particular response to a specific input (Loke 1999). Learning is accomplished by using a so called "training algorithm", that is a mathematical formulation of the rules that determine the magnitude of weight adjustment. If properly trained, the ANN is able to generalize from the examples used for training.

A static back-propagation ANN was trained on 6 months of 1-hour sample-time WWTP input-output data. The training data was generated with the help of the mechanistic WWTP model. The ANN used in this study is a static backpropagation network. This type of network is one of the simplest: a modeler with no experience in ANNs would most likely start with such type of ANN. Due to the relatively easy model building and training procedure, the comprehensive description provided in almost every ANN textbook, as well as their good overall generalization capabilities, the backpropagation networks are very popular.

The main characteristics of the used ANN are shown in Table 1. These ANN settings enable good prediction performance and fast training, and are in accordance with the guidelines provided by Ráduly (2005).

Table 1. The ANN Configuration Used in the Study

$\begin{array}{ll}\text { ANN type } & \text { Static, feedforward } \\ \text { Training type } & \text { Backpropagation } \\ \text { Training algorithm } & \text { Scaled conjugate gradient algorithm } \\ \text { Initial weights } & \text { Random Gaussian distribution } \\ \text { Stopping criterion } & \text { Cross-validation } \\ \text { No. of neurons } & 152 / 35 / 13 / 13 \\ \text { Neuron types } & \text { Tan-sig / Tan-sig / Tan-sig / Linear } \\ \text { Input window size } & \text { 15 lagged values covering 20 h } \\ \text { Data processing } & \text { Zero-mean normalization [-1,+1] } \\ \text { Validation } & \text { every } 7^{\text {th }} \text { sample used for validation }\end{array}$




\section{RESULTS AND DISCUSSION}

\section{Prediction Accuracy}

The monthly COD loads to the river predicted by the two WWTP models for the one year test period are presented in Figure 2. As can be seen, the results are very similar; the largest difference between the two predictions is in July (5.4\% relative error). This can be explained by a holiday effect, which is included in the influent data and leads to reduced pollutant loads for this period: since the ANN was not trained on such low loads, it overestimates the pollutant concentrations in the effluent during the holiday period. Unlike in July, relative errors stay below $2.4 \%$ for the rest of the year.

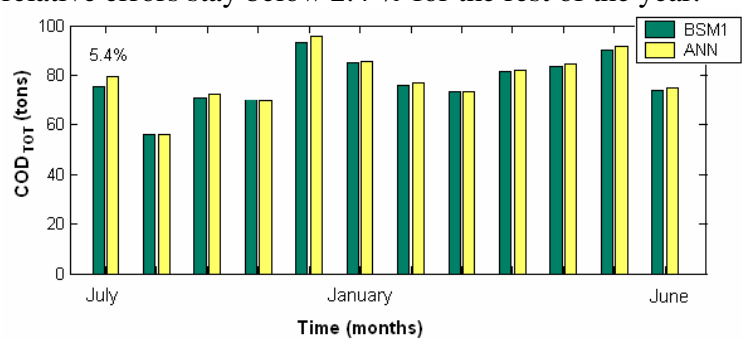

Figure 2. Monthly COD Loads Predicted by the BSM1 and the ANN Model.

In order to verify how ANN imprecision influences the predicted pollutant concentrations in the river, the three most important indicators were assessed: $\mathrm{NH}_{4}, \mathrm{DO}$ and $\mathrm{BOD}_{5}$. The results are compared on concentration-time graphs in river reaches no. 7 (WWTP discharge point) and no. 10 (3 km downstream). The period selected for illustration covers 15 days in the end of June (days 594609 ), with a rain event on days 598-600.
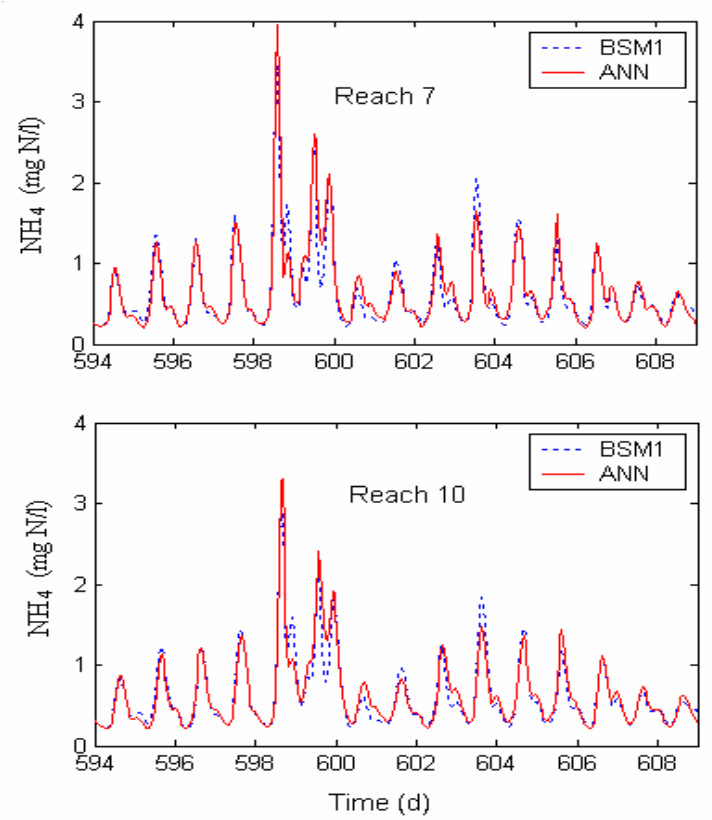

Figure 3. Effect of BSM1 vs. ANN Prediction on the $\mathrm{NH}_{4}$ Concentration in River Reaches no. 7 and 10
Figure 3 illustrates the $\mathrm{NH}_{4}$ concentration in the two selected river reaches. As the figure shows, in reach no. 7 he ammonia peak is a bit overestimated $(4 \mathrm{mg} / \mathrm{l}$ instead of $3.5 \mathrm{mg} / \mathrm{l}$ ) when the ANN is used instead of the BSM1 in the integrated model. For the rest of the period the predicted concentrations are very similar. The situation is more or less the same $3 \mathrm{~km}$ downstream, in reach no. 10: the ammonia peak is about $10 \%$ overestimated when the ANN is used.

Figure 4 illustrates the DO concentrations in river reaches 7 and 10 . The oscillation of the DO concentration is partly due to the changes in biodegradable pollutant loads discharged from the WWTP. However, the main contribution is due to the photosynthetic activity in the river. Oxygen depletion on day 599 can be observed with a minimum of $4.5 \mathrm{mg} / \mathrm{l}$ DO in reach no. 7. The DO concentrations in the river reaches investigated are very similar for the BSM1 and ANN predictions; no significant differences can be observed.
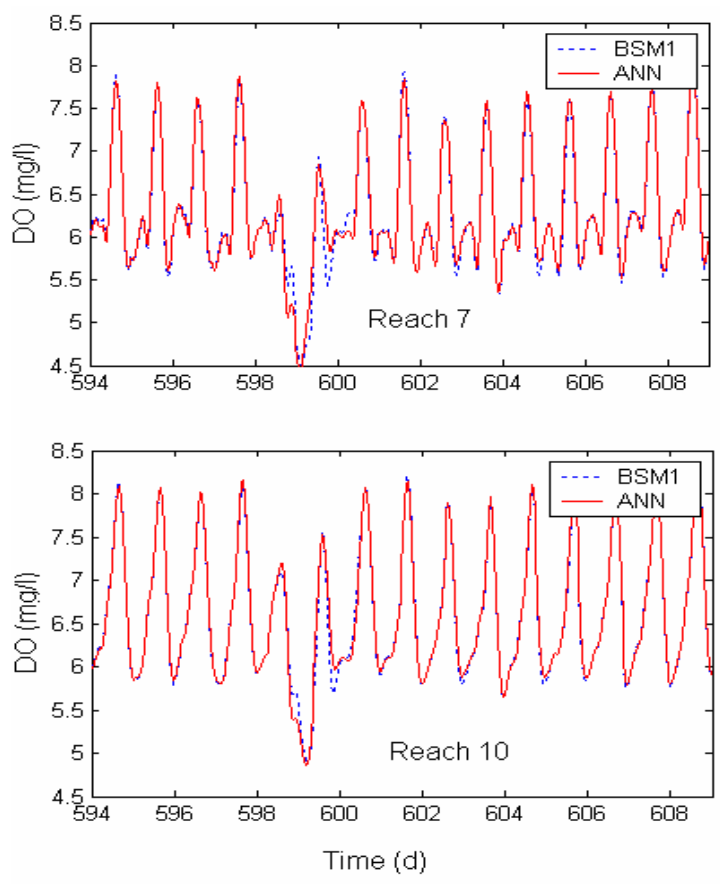

Figure 4. DO in the River in the Case of BSM1 and ANN Predictions

The last river quality indicator evaluated is the $\mathrm{BOD}_{5}$ (Fig. 5). There is a small $\mathrm{BOD}_{5}$ peak on days 598-600, when the $\mathrm{BOD}_{5}$ values are $\sim 1 \mathrm{mg} / \mathrm{l}$ higher than under dry weather conditions. The dry-weather predictions are practically the same in the case of BSM1 and ANN predictions. During the $\mathrm{BOD}_{5}$ peak the results are slightly overestimated when the ANN model is used. However, the differences are small; the relative error does not exceed $3 \%$ even in this period. 

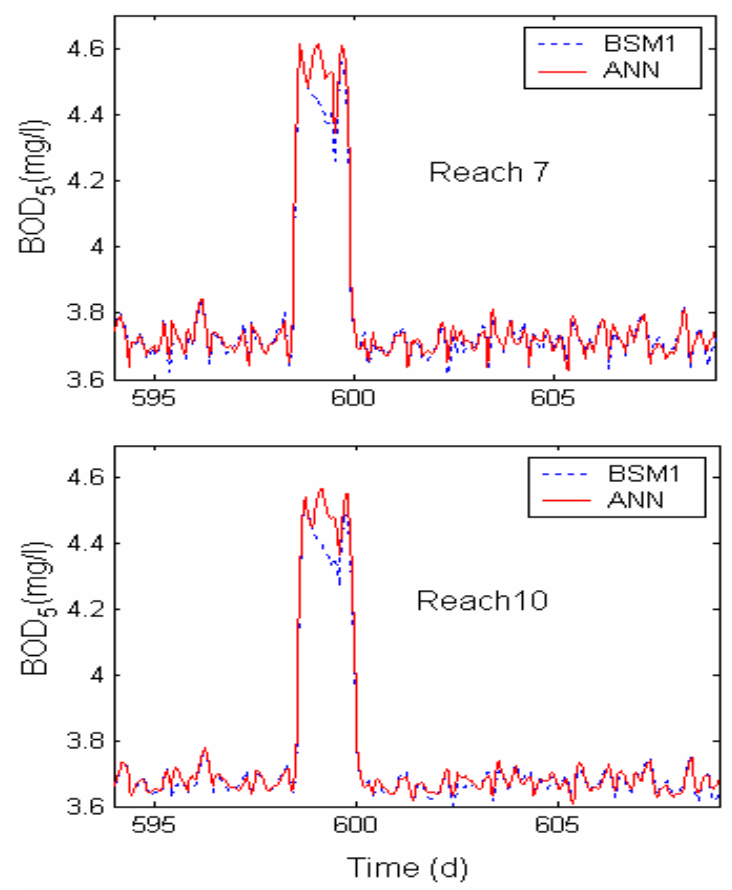

Figure 5. $\mathrm{BOD}_{5}$ Concentrations in the River in the case of BSM1 and ANN Predictions

\section{Comparison of Simulation Speeds}

The simulation speed of the ANN model is several orders of magnitude higher than that of the mechanistic WWTP model. However, in an integrated model the simulation speed of the other submodels might be a limiting factor. Thus, for a fair comparison, the simulation speed of the integrated models is assessed. The time needed for simulating the urban wastewater system over one year (dynamic influent data) is shown in Table 2. In the case of the configuration using the ANN model of the WWTP, the time used for ANN training and for the generation of training data is also shown. The integrated model using the ANN shows to be $\sim 23$ times faster than the other configuration. If training- and data generation time is also added to simulation time, this factor is only 1.2 (calculating with 1 year simulation and 6 moths of training data; for longer simulations this factor will be considerably higher). That indicates that even for the case where the WWTP model is modified (and thus the response of the WWTP model on the input is different), re-building the ANN and performing the simulation takes less time than running of the same simulation with the mechanistic integrated model. Moreover, once a trained ANN is available, the WWTP simulation can be repeated very fast, since no new ANN training is needed for each new simulation. This opens for example possibilities to use simulation of the UWWS to rapidly compare the usefulness of different real-time control strategies applied to the sewer system.
Table 2. CPU Time (minutes) Needed for Running a 1year Simulation (AMD A64 2800+ Desktop)

\begin{tabular}{|c|l|c|c|}
\hline \multicolumn{2}{|c|}{ Integrated UWWS model using } & BSM1 & ANN \\
\hline \multirow{2}{*}{$\begin{array}{c}\text { CPU } \\
\text { time } \\
(\text { min })\end{array}$} & Simulation & 21 & 0.9 \\
\cline { 2 - 4 } & Data generation & - & 10 \\
\cline { 2 - 4 } & Training & - & 6 \\
\cline { 2 - 4 } & Total & 21 & 16.9 \\
\hline
\end{tabular}

\section{CONCLUSIONS}

The results of the experimentation with the ANN in an integrated modeling environment are very promising. The substitution of the WWTP model with the ANN is very straightforward and does not pose any special problem. The prediction errors, caused by the ANN imprecision, do not significantly alter the simulated river conditions (in the case of the three river quality indicators tested in this study). The prediction errors are of an acceptable level in integrated-scale modeling application, considering the large uncertainties of model inputs and process kinetics when modeling an UWWS. The ANN model catches well the model dynamics, and pollutant peaks are clearly visible both for $\mathrm{NH}_{4}$ and for $\mathrm{BOD}_{5}$. The oxygen depletion is also correctly predicted, both as magnitude and as spatial and temporal distribution in the river. The monthly pollutant loads predicted by the ANN model of the WWTP are also very close to BSM1 predictions, except for July, when the holiday effect is not simulated correctly. This confirms that the holiday period should be included in the training data. The simulation speed of the integrated model is 23 times higher when the ANN is used instead of the BSM1, meaning that an optimization study of the integrated UWWS that would normally need 2 days of simulation can now be performed in about 2 hours. This justifies the use of the proposed model configuration for long-term simulations of the integrated UWWS.

\section{FURTHER RESEARCH}

Future work to be done concerns the prediction accuracy of the ANNs, and the application of this model reduction strategy to other cases. Better results might be expected from recurrent networks with external feedback; these networks are more suitable for the representation of discrete dynamic systems. Such a neural network could, for example, use the biomass concentrations in the WWTP as feedback (i.e. the biomass concentrations would be used as input in the next time-step). In future studies an ANN model of the sewer system could be used, for example, if no internal variable is of interest and only the input/output response is important. On the integrated modeling scale, river segments could also be modeled with ANNs; having a high simulation speed, a large number of such river 
segments could be used, and thus the flow and mixing in the river could be modeled in a more detailed way.

\section{REFERENCES}

Benedetti L., Meirlaen J. and Vanrolleghem P.A. 2004. Model connectors for integrated simulations of urban wastewater systems. Sewer Networks and Processes within Urban Water Systems. J.-L. Bertrand-Krajewski, M. Almeida, J. Matos and S. Abdul-Talib (eds.), Water and Environment Management Series (WEMS), IWA Publishing, pp. 13-21.

Butler D. and Schütze M. (2005) Integrating simulation models with a view to optimal control of urban wastewater systems. Environmental Modelling and Software, 20(4), 415-426.

Copp J.B. (ed.) 2002. The COST Simulation Benchmark Description and Simulator Manual. ISBN 92-894-1658-0, Office for Official Publications of the European Communities, Luxembourg.

Gernaey K.V., Rosen C., Benedetti L. and Jeppsson U. (2005) Phenomenological modelling of wastewater treatment plant influent disturbance scenarios. Proc. $10^{\text {th }}$ International Conference on Urban Drainage, August 2126, Copenhagen (Denmark).

Gernaey K.V., Rosen C. and Jeppsson U. (2006). WWTP dynamic disturbance modelling - an essential module for long-term benchmarking development, Water Science and Technology, 53(4-5), 225-234.

Haykin, S. (1994). Neural Networks. Macmillan College Publishing Company, Inc, New York.

Henze M., Grady C.P.L., Gujer W., Marais G.v.R and Matsuo T. (1987) Activated Sludge Model No.1. IAWPRC Scientific and Technical Report No.1, IAWPRC, London. ISSN: 1010-707X

Jeppsson, U., Rosen, C., Alex, J., Copp, J., Gernaey, K.V., Pons, M.-N. and Vanrolleghem, P.A. (2006) Towards a benchmark simulation model for plant-wide control strategy performance evaluation of WWTPs, Water Science and Technology, 53(1), 287-295.

Loke E. 1999 Artificial neural networks in urban storm drainage. PhD thesis, Dept. of Environmental Science and Engineering, Technical University of Denmark, Lyngby, Denmark.

Ráduly B. 2005. Artificial neural network applications in the urban water quality modeling. $\mathrm{PhD}$ thesis, Department of Hydraulic and Environ. Engineering, University of Pavia, Pavia, Italy.

Reichert P., Borchardt D., Henze M., Rauch W., Shanahan P., Somlyódy L. and Vanrolleghem P. (2001) River Water Quality Model No. 1. Scientific and Technical Report No 12. IWA Publishing, London, UK.

Schütze M.R., Butler D. and Beck M.B. (2002) Modelling, simulation and control of urban wastewater systems. London, Springer, ISBN: 1-8523-3553-X

Takács I., Patry G.G. and Nolasco D. (1991) A dynamic model of the clarification thickening process. Water Research, 25(10), 1263-1271.

Vanrolleghem P.A., Benedetti L. and Meirlaen J. (2005) Modelling and real-time control of the integrated urban wastewater system. Environmental Modelling and Software, 20(4), 427-442.

\section{AUTHOR BIOGRAPHIES}

BOTOND RÁDULY was born in M-Ciuc (Romania) in 1978 and is graduated in Information Technology of Chemical Systems at the Babes-Bolyai University of Cluj Napoca, where he obtained his degree in 2001. He was research assistant for a short time at the Central Nuclear Research Institute in Budapest, (Hungary). He completed his $\mathrm{PhD}$ in 2006 at the University of Pavia, Italy, in the field of wastewater treatment plant modeling.

KRIST V. GERNAEY was born in Ghent (Belgium), in 1970. He obtained a Masters in Chemistry and Agricultural Industries in 1993 and a PhD in Applied Biological Sciences in 1997, both from Ghent University. After completing his $\mathrm{PhD}$, he has been a postdoctoral fellow in Belgium (Ghent University, Faculty of Applied Biological Sciences), Canada (Montreal, École Polytechnique de Montréal), Denmark (Lyngby, Technical University of Denmark) and Sweden (Lund, Lund University). He was recently appointed as associate professor at the Technical University of Denmark, where he works at the Center for Biochemical Engineering (Dept. of Chemical Engineering). His research interests include modelling (first principles and data-driven) applied to biological systems, process control, systems biology and biochemical engineering.

ANDREA GIUSEPPE CAPODAGLIO. Professor of Sanitary and Environmental Engineering at the University of Pavia. Visiting Researcher at the Water Resaerch Centre of the Italian National Research Council, Adjunct Professor at Marquette University (Milwaukee, USA). Graduated cum laude in Civil Engineering from the University of Pavia, $\mathrm{PhD}$ in Water Resources and Environmental Engineering at Marquette University. Teaching and research interests are mainly in the field of environmental quality and modelling, diffusion and transport of contaminants in the environment.

ERIK LINDBLOM was born in Gothenburg (Sweden) in 1978. He obtained a Masters in Environmental Engineering from the Lund Insitute of Technology (Lund University) in 2003. He is know enrolled in a $\mathrm{PhD}$ program at the Institute of Environment \& Resources (Technical University of Denmark) in the field of integrated urban wastewater system modelling. 